\title{
Rainfall downscaling and flood forecasting: a case study in the Mediterranean area
}

\author{
N. Rebora ${ }^{1}$, L. Ferraris ${ }^{1,2}$, J. von Hardenberg ${ }^{3,1}$, and A. Provenzale ${ }^{3,1}$ \\ ${ }^{1}$ CIMA, University of Genoa and University of Basilicata, Savona, Italy \\ ${ }^{2}$ DIST, University of Genoa, Genoa, Italy \\ ${ }^{3}$ ISAC-CNR, Turin, Italy
}

Received: 31 October 2005 - Revised: 29 May 2006 - Accepted: 29 May 2006 - Published: 12 July 2006

\begin{abstract}
The prediction of the small-scale spatial-temporal pattern of intense rainfall events is crucial for flood risk assessment in small catchments and urban areas. In the absence of a full deterministic modelling of small-scale rainfall, it is common practice to resort to the use of stochastic downscaling models to generate ensemble rainfall predictions to be used as inputs to rainfall-runoff models. In this work we present an application of a new spatial-temporal downscaling procedure, called RainFARM, to an intense precipitation event predicted by the limited-area meteorological model Lokal Model over north-west Italy. The uncertainty in flood prediction associated with the small unresolved scales of forecasted precipitation fields is evaluated by using an ensemble of downscaled fields to drive a semi-distributed rainfall-runoff model.
\end{abstract}

\section{Introduction}

In the Mediterranean region many cities are located in floodprone areas and millions of people are exposed to inundation risk. For this reason issuing early flood warning to the population is crucial to avoid loss of lives and to reduce property damage.

The solution to this problem relies on the knowledge of meteorological and hydrological processes that lead to flood formation; depending on the characteristics of the watershed, many different approaches that use meteorological and hydrological models as well as observations have been proposed (Droegemeier et al., 2000). Very large basins, with areas greater than $10000 \mathrm{~km}^{2}$, have concentration times larger than $24 \mathrm{~h}$; in this case flood forecasting based on precipitation observations coupled with hydrological models suffices. However, small and medium catchments, with areas smaller

Correspondence to: N. Rebora

(nicola@cima.unige.it) than $1000 \mathrm{~km}^{2}$, typical for the Mediterranean environment, have concentration times of less than $12 \mathrm{~h}$; as this is less than the time necessary for the population to react by following alert procedures (Siccardi et al., 2005), rainfall forecasts at these scales, or smaller, become crucial. The use of limitedarea meteorological models (LAMs) that provide precipitation forecasts on scales of about $100 \mathrm{~km}^{2}$ and a few hours is a common approach to this issue (Lin et al., 1985; Bacchi et al., 2003). However, obtaining reliable predictions from numerical models at these resolutions is still difficult. An alternate approach is to use statistical techniques to downscale modeled precipitation to the fine resolutions needed for hydrological applications (Droegemeier et al., 2000; Ferraris et al., 2002; Siccardi et al., 2005).

A downscaling procedure consists of a stochastic algorithm that allows for generating an ensemble of possible realizations of the small-scale rainfall field starting from a smoother field predicted on larger scales. The precipitation fields generated by this approach are required to satisfy large-scale constraints imposed by the meteorological forecast (e.g., the total rainfall volume) and should be consistent with the known statistical properties of the small-scale rainfall distribution.

Here we consider a simple hydrometeorological flood forecasting chain composed of three elements: (a) Limited Area Model precipitation predictions, (b) ensembles of high-resolution rainfall fields generated by a downscaling algorithm and (c) ensembles of peak discharges obtained by coupling the rainfall downscaling ensembles with a semidistributed rainfall-runoff model. The output of this procedure is a probabilistic distribution of peak discharges whose variability is generated by the small-scale fluctuations of the precipitation input provided by the downscaling procedure.

The aim of this work is to show the performance of a downscaling algorithm, designed to generate smallscale rain rate fluctuations, that preserves the spatialtemporal evolution of rainfall patterns predicted by a LAM

Published by Copernicus GmbH on behalf of the European Geosciences Union. 


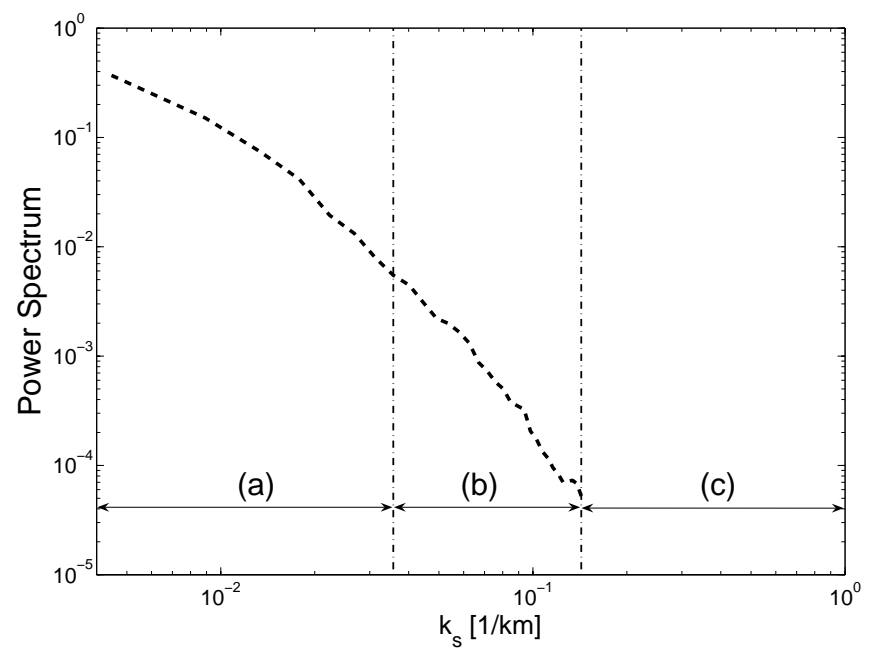

Fig. 1. Example of the possible scales range of a power spectrum obtained from the spatial analysis of a LAM prediction: (a) reliable scales, (b) unreliable scales and (c) unresolved scales.

(Rebora et al., 2005, 2006). Here we consider only the ability of the downscaling procedure to generate, starting from a single meteorological forecast, an ensemble of highresolution precipitation fields that in turn lead to an ensemble of possible hydrographs. A comparison between observed and forecasted hydrographs will be reported elsewhere.

The work is organized as follows: a brief description of the downscaling procedure is given in the next section. In the third section we discuss an example implementation of the hydrometeorological chain. We present an application of the downscaling model and we generate an ensemble of possible high-resolution rain fields. These fields are then used as inputs to a semi-distributed rainfall-runoff model. A discussion and conclusions are presented in Sect. 4.

\section{The RainFARM downscaling procedure}

A hydrometeorological forecasting chain designed for operational purposes requires robust and computationally fast downscaling models. Many procedures have been proposed for rainfall downscaling to this date. These algorithms can be grouped into three main families: (1) multifractal cascades, (2) non-linearly transformed autoregressive models, and (3) processes based on the superposition of many rainfall cells (cluster models). All these models have been proven to score fairly well in reproducing the observed small-scale statistical properties of precipitation (Ferraris et al., 2003b). However, linking these models with the features of the large scale fields is not immediate. Many downscaling procedures currently available for operational purposes account only for the total precipitation predicted by the LAM over a given spatialtemporal domain; some other models are based on CAPE (Convective Available Potential Energy) predicted by me-

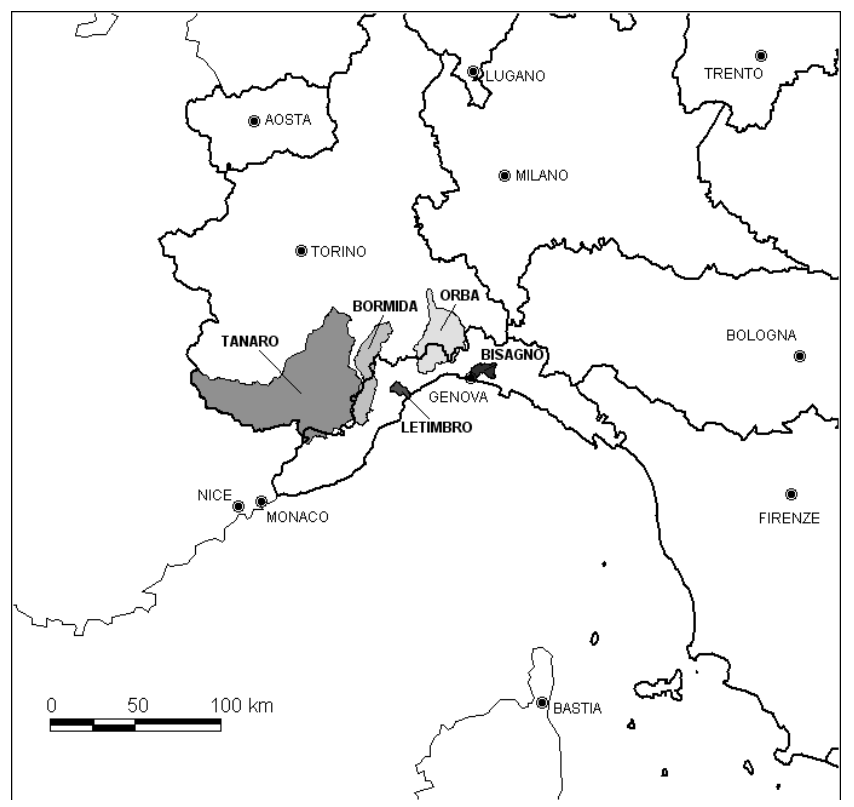

Fig. 2. The downscaling domain over North-Western Italy and the catchments considered in the work.

teorological models (Perica and Foufoula-Georgiou, 1996; Venugopal et al., 1999), but other information deriving from meteorological predictions is often not preserved. For example, the localization in space and in time of large scale structures in the rainfall field, at scales reliably predicted by the model, can be crucial for predicting sudden floods in small catchments and in urban areas (Droegemeier et al., 2000).

In this work a new downscaling procedure is used. This approach is able to account for the reliable features of the meteorological prediction and its parameters can be directly derived from the large-scale field with no need for calibration. This procedure is called RainFARM, Rainfall Filtered AutoRegressive Model, and it was proposed by Rebora et al. (2006) to which we refer for a complete description and further details. RainFARM belongs to the family of algorithms called metagaussian models (see, e.g. Guillot and Lebel 1999) and it is based on a nonlinear transformation of a linearly correlated process. This approach is closely related to the Turning Bands Method (Matheron, 1973) and has been used both for satellite-based rainfall measurement validation and for stochastic rainfall modelling (Bell and Kundu, 2003; Bell, 1987; Lanza, 2000). The model is able to generate small-scale rainfall fields that take into account not only the total amount of precipitation predicted by the meteorological model but also its linear correlation structure and the position of the main rainfall patterns. Due to the straightforward link between the model parameters and the large-scale field, this model is suitable for operational downscaling procedures.

RainFARM uses the spectral information of large-scale meteorological predictions and generates fine resolution precipitation fields by propagating this information to smaller 


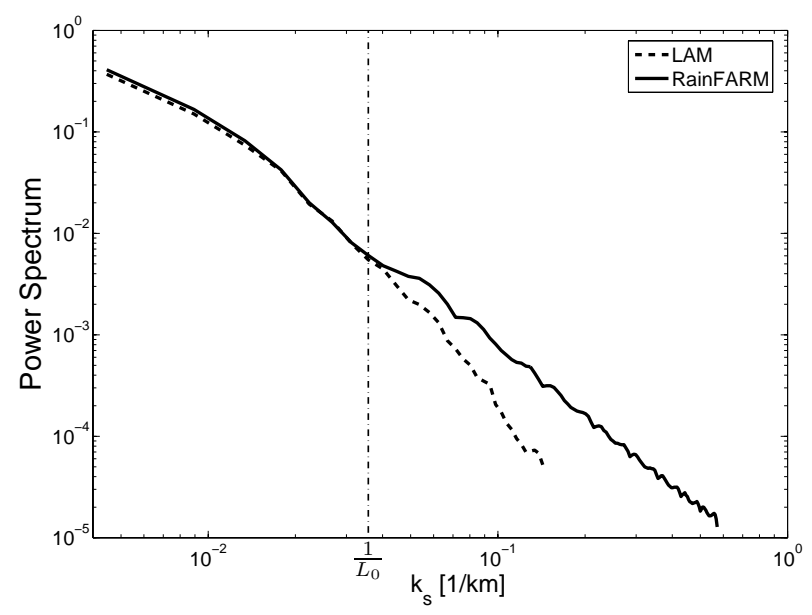

Fig. 3. Spatial power spectrum of the original LAM field (dashed line) compared to that obtained for a downscaled field (solid line).

scales. The basic idea is to reconstruct the Fourier spectrum of the small-scale precipitation field by preserving the LAM information at the scales where we are confident in the meteorological prediction. The rainfall field is seen as the superposition of a finite number of harmonics with amplitudes decreasing as spatial and temporal scales become smaller. For a given realization (the predicted field at hand) the harmonics at large scales are assumed to be well predicted by the meteorological model, and should be preserved. However, when going down to smaller scales, they are less and less reliable and can be perturbed or replaced by harmonics whose properties respect the statistical properties at small scales of such rainfall fields. This gives a set of realizations which displays a range of uncertainty which is linked to the effect of the scales poorly resolved by the model.

A major concern is to figure out which are the scales below which the forecasted fields are considered to be unreliable. Their definition depends on the meteorological model we are downscaling and it is related to the predictability of the meteorological scenario we are considering. It is well known that due to numerical diffusion, a meteorological model is not reliable at scales smaller than six to four times its resolution (Patterson and Orszag, 1971). For these reasons we define three different scale regimes (Fig. 1): (a) reliable scales, where the information should be preserved by the downscaling procedure, (b) unreliable scales, i.e. scales numerically resolved by the model but unreliable due to numerical issues or to the lack of assimilation procedures; (c) unresolved scales, which are scales not resolved by the model but which are needed for hydrological purposes.

The transition from reliable to unreliable scales depends on model characteristics, such as the resolution of the network used for data assimilation, the forecast lead time and the type of meteorological conditions (e.g., convective vs. stratiform).
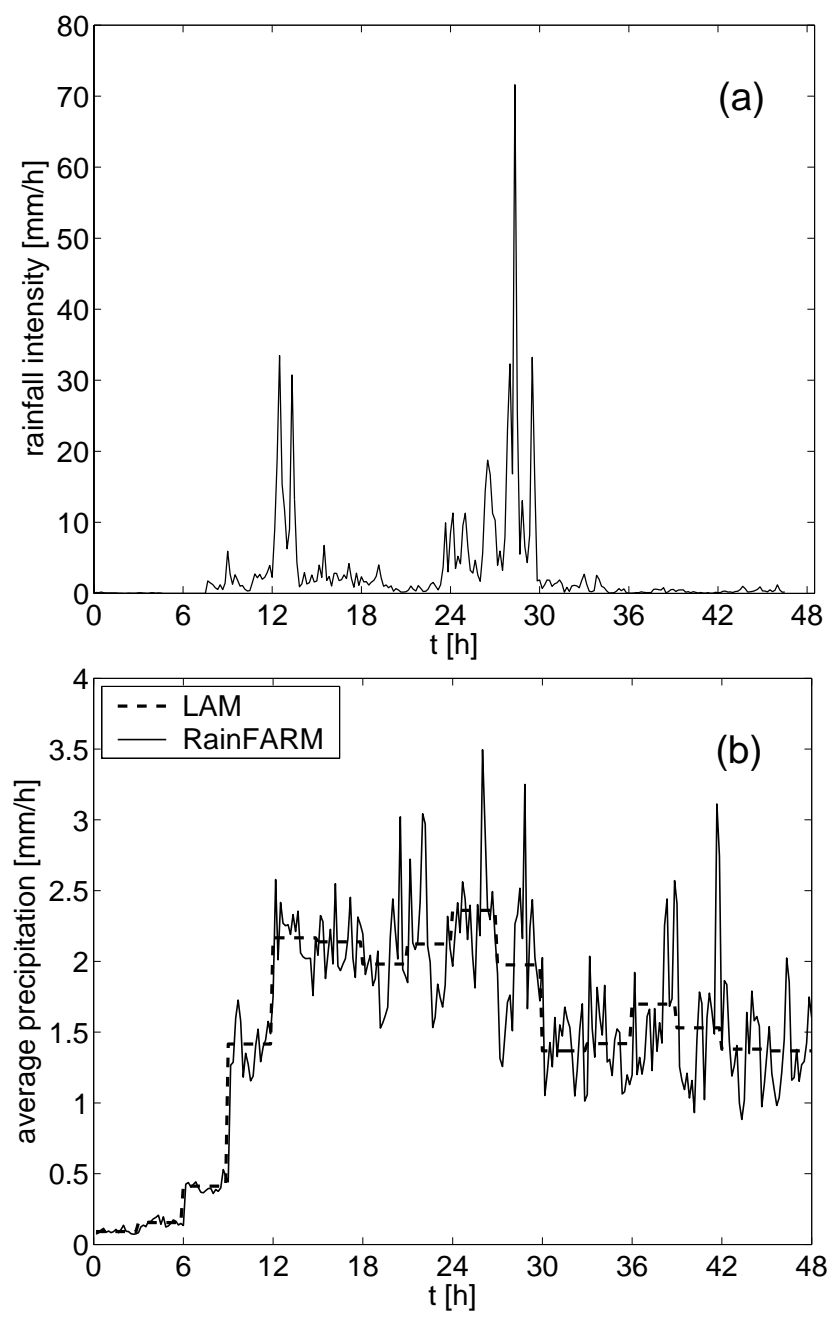

Fig. 4. Panel (a): Evolution on a single continental pixel of precipitation downscaled to $10 \mathrm{~min}$ resolution. Panel (b): Temporal evolution of the instantaneous spatial average of the large-scale LAM field (dashed line) and of one realization of the stochastic field (solid line).

The RainFARM has four free parameters, named $L_{0}, T_{0}$, $\alpha$ and $\beta . L_{0}$ and $T_{0}$ represent the spatial and temporal resolutions at which the precipitation prediction is considered reliable and they should be fixed by the user depending on the numerical model considered ${ }^{1}$. The spectral parameters $\alpha$ and $\beta$ are estimated in real-time from the LAM power spectrum.

The RainFARM works as follows. The aggregation of the LAM field on spatial and temporal scales $L_{0}$ and $T_{0}$, generates the starting field, called $P$. From this coarse-grained

\footnotetext{
${ }^{1}$ The spectral parameters and the reliable scales depend also on the synoptic weather pattern at hand. In this work we assume that it is possible to estimate them directly from the large scale behavior of the LAM fields. The alternative and more complex exercise of conditioning them on synoptic patterns will be explored in the future.
} 


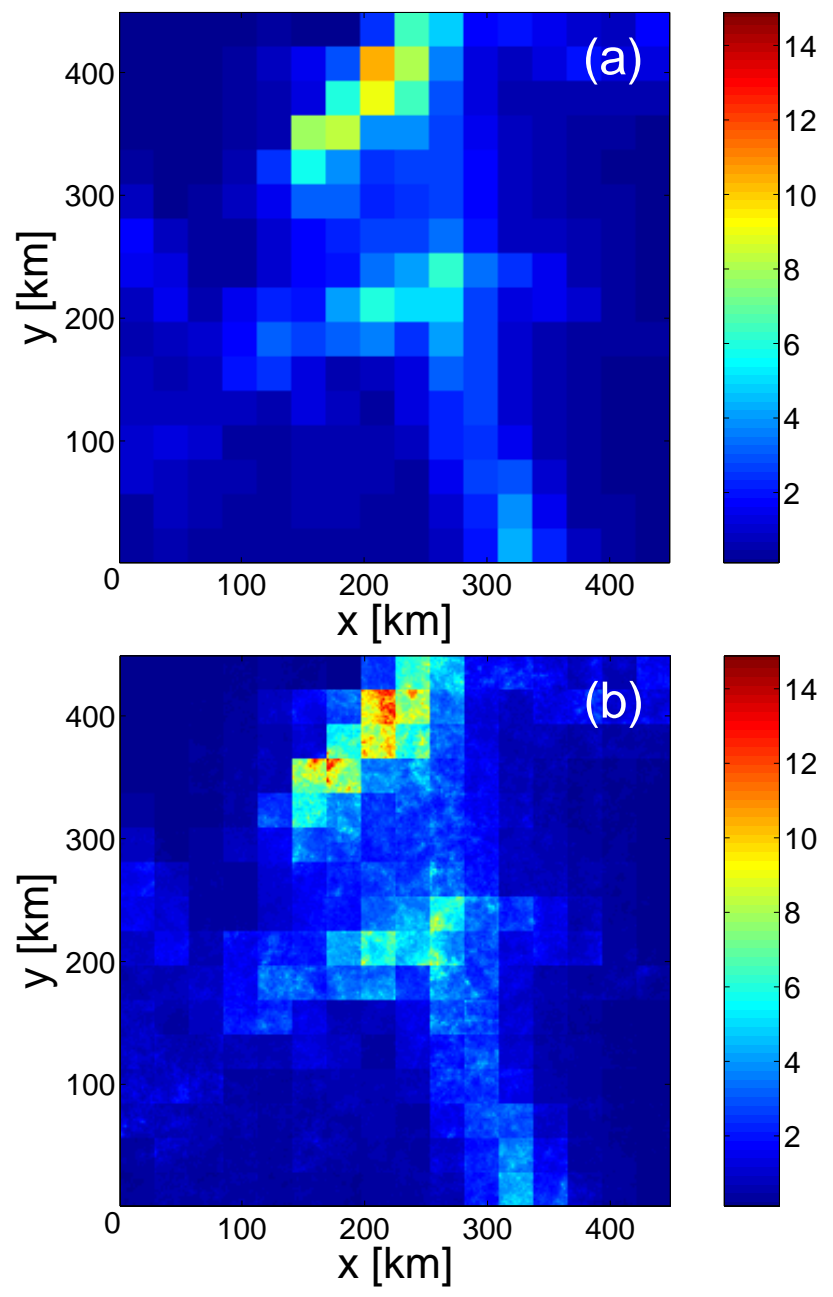

Fig. 5. Time average of the original, LAM field panel (a) and of one realization of the stochastic field generated by the RainFARM panel (b). The values indicate the average precipitation in $\mathrm{mm} / \mathrm{h}$.

prediction RainFARM produces downscaled fields by following these steps:

1. Computation of the Fourier transform of the coarsegrained LAM-predicted field $\mathrm{P}(\mathrm{X}, \mathrm{Y}, \mathrm{T})$. This procedure generates a spatial-temporal Fourier spectrum $\hat{P}\left(K_{X}, K_{Y}, \Omega\right)$ defined as follows:

$\hat{P}\left(K_{X}, K_{Y}, \Omega\right)=$

$\sum_{X=0}^{D_{X}} \sum_{Y=0}^{D_{Y}} \sum_{T=0}^{D_{T}} P(X, Y, T) e^{-i\left(X K_{x}+Y K_{Y}+T \Omega\right)}$

Where $D_{X}, D_{Y}, D_{T}$ are the domain sizes in space and time respectively (Press et al., 1992). Here, $K_{X}$ and $K_{Y}$ are the wavenumbers in the $X$ and $Y$ directions and $\Omega$ is the angular frequency. Clearly, $\left(K_{X}, K_{Y}\right) \leq \pi / L_{0}$ and $\Omega \leq \pi / T_{0}$, where $\pi / L_{0}$ is the Nyquist wavenumber and $\pi / T_{0}$ is the Nyquist frequency of the field to be downscaled.

2. Estimate of the space-time power spectrum $\left|\hat{P}\left(K_{X}, K_{Y}, \Omega\right)\right|^{2}$ of the aggregated LAM field $P$ as the square of the modulus of its Fourier transform $\hat{P}$.

3. Extrapolation of the power spectrum $|\hat{P}|^{2}$ to small scales. To do so, we assume the power spectrum of the rainfall field to have an approximate power-law behavior, consistent with the outcome of several analyses on the structure of precipitation fields (e.g. Crane (1990); Menabde et al. (1999); Ferraris et al. (2003a,b)). The procedure estimates the spatial and temporal logarithmic slopes ( $\alpha$ and $\beta$ respectively) of the LAM power spectrum, $|\hat{P}|$. For simplicity, we assume isotropy in the two spatial directions ${ }^{2}$.

4. Generation of a Fourier spectrum $\hat{g}\left(k_{x}, k_{y}, \omega\right)$, defined as

$\hat{g}\left(k_{x}, k_{y}, \omega\right)=\left|\hat{g}\left(k_{x}, k_{y}, \omega\right)\right| \exp (i \phi)$

where $\phi\left(k_{x}, k_{y}, \omega\right)$ are random, uniformly distributed phases. The wavenumbers $\left(k_{x}\right.$ and $\left.k_{y}\right)$ and the frequency $(\omega)$ range from the scales corresponding to the downscaling domain size to those associated with the downscaling resolution. We use the functional form

$\left|\hat{g}\left(k_{x}, k_{y}, \omega\right)\right|^{2}=\left(k_{x}^{2}+k_{y}^{2}\right)^{-\alpha / 2} \omega^{-\beta}$

By inverting the Fourier spectrum, $\hat{g}$, we obtain a Gaussian field $g(x, y, t)$ defined on the whole range of scales between the domain size and the downscaling resolution, which we normalize to unit variance.

5. Generation of a synthetic precipitation field, $\tilde{r}(x, y, t)$, by taking a nonlinear transformation of the Gaussian field $g$. Here we use the simple transformation

$\tilde{r}(x, y, t)=\exp [g(x, y, t)]$

which leads to a log-normal field $\tilde{r}$.

6. We force the synthetic field to be equal to the original field $P$ when aggregated on the scales $\left(L_{0}, T_{0}\right)$ by defining a new field:

$$
r(x, y, t)=\tilde{r}(x, y, t) \frac{P(X, Y, T)}{\tilde{R}(X, Y, T)}
$$

\footnotetext{
${ }^{2}$ Since the RainFarm algorithm preserves the structure of the LAM field at large scales, we only impose isotropy at the unresolved scales.
} 

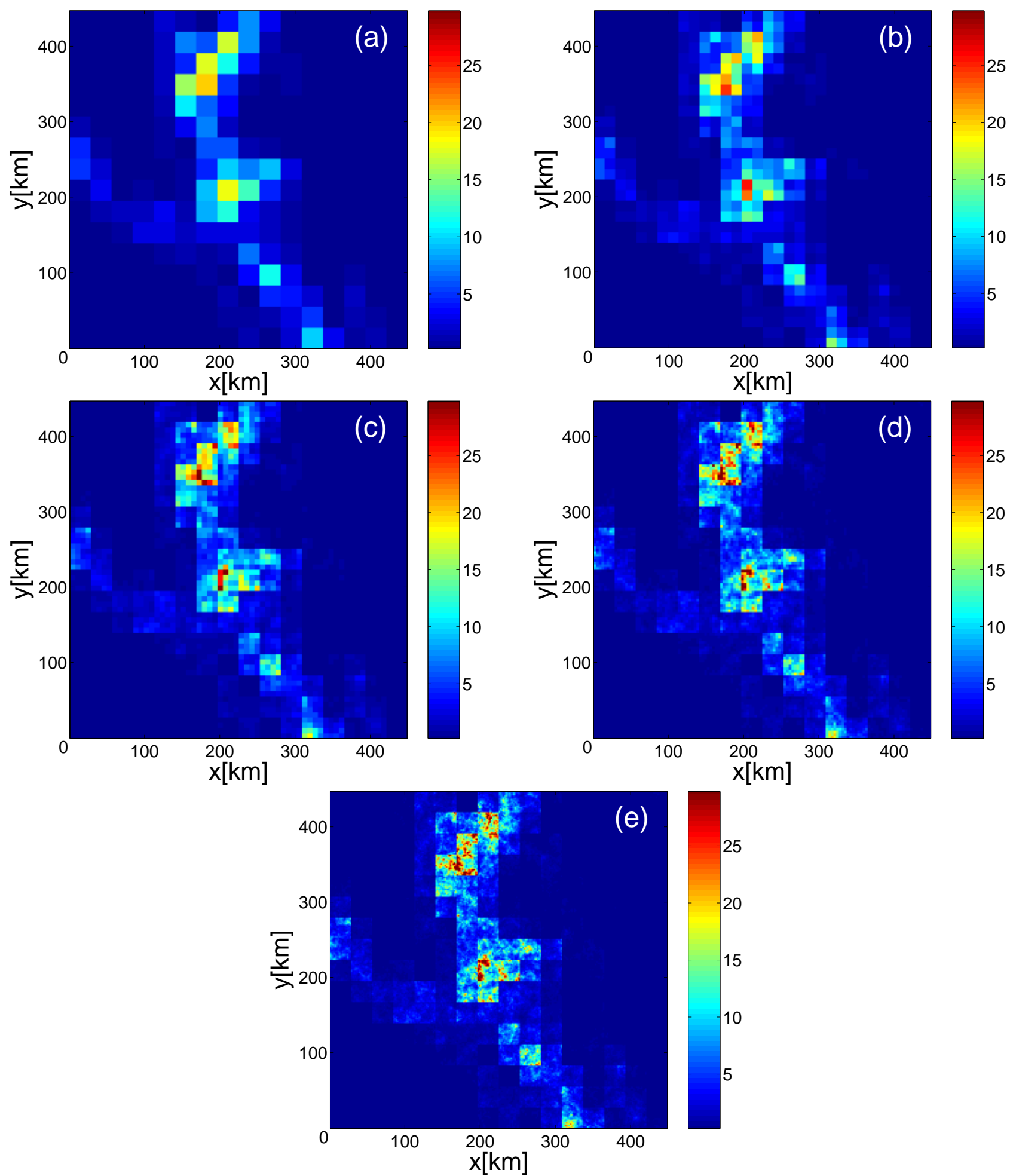

Fig. 6. Example of spatial downscaling. The LAM field panel (a) indicates the rainfall predicted from $t=33 \mathrm{~h}$ to $t=36 \mathrm{~h}$ at the spatial reliable resolution of $28 \mathrm{~km}$. The rainfall intensity fields generated by RainFARM at different scales panels (b)-(e) show the fine-scale precipitation at the same time interval but at different spatial resolutions: (b) $14 \mathrm{~km}$, (c) $7 \mathrm{~km}$, (d) $3.5 \mathrm{~km}$ and (e) $1.75 \mathrm{~km}$.

Where $\tilde{R}$ represents the field $\tilde{r}$ aggregated at the scale $\left(L_{0}\right.$, $\left.T_{0}\right)$. When aggregated on space and time scales larger or equal to $L_{0}$ and to $T_{0}$, the field $r$ behaves exactly as the original field $P$. The stochastic nature of the downscaled field 


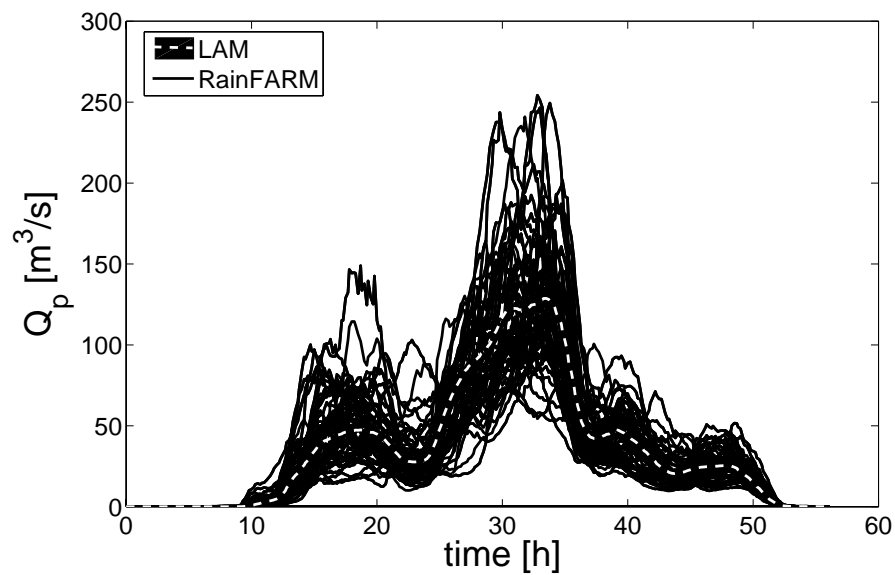

Fig. 7. Ensemble of 100 hydrographs obtained for the Bisagno catchment $\left(A \simeq 100 \mathrm{~km}^{2}\right.$ ). The white line represent the deterministic hydrograph obtained by using the LAM prediction as an input for the rainfall-runoff model.

$r$ is associated with the choice of the set of random Fourier phases. By choosing different sets of random Fourier phases, one can generate a large number of stochastic fields which are all equal to $P$ when aggregated on space and time scales larger than $L_{0}$ and $T_{0}$, and which are different, but with similar statistical properties, on smaller scales.

\section{The hydrometeorological forecasting chain}

Here we consider a simple procedure that will lead us to issue a probabilistic flood forecast by starting from a single deterministic precipitation prediction. This approach integrates LAM predictions, stochastic downscaling and rainfall-runoff modelling.

We consider an intense rainfall event forecasted by the Lokal Model (Deutscher Wetterdienst) over North-Western Italy. This event was predicted on 30 October 2004 starting from 00:00 UTC and has a total duration of $48 \mathrm{~h}$. The forecast has a spatial resolution of $7 \mathrm{~km}$ by $7 \mathrm{~km}$ and a time resolution of $3 \mathrm{~h}$.

We define the downscaling domain as a square area of side $448 \mathrm{~km}$ that contains the Liguria, Lombardia, Piemonte and Valle d'Aosta regions in Italy (Fig.2). An ensemble of 100 fields is generated by RainFARM starting from the LAM prediction over the dowscaling domain. These fields have a resolution of $1.75 \mathrm{~km}$ by $1.75 \mathrm{~km}$ in space and $10 \mathrm{~min}$ in time.

The high-resolution precipitation ensemble is then coupled with a semi-distributed rainfall-runoff model. This will lead to the generation of a hydrological ensemble forecast in several selected catchments within the downscaling domain.

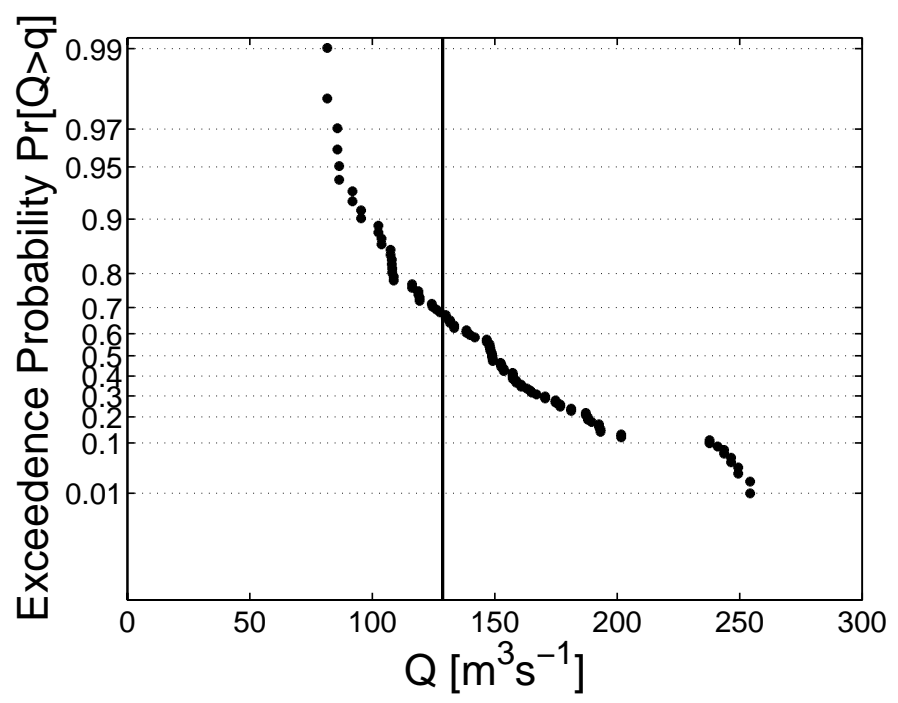

Fig. 8. Peak discharge exceedence probability plotted on a Gumbel chart for the Bisagno River. The black points represent peak discharge data generated by the downscaled ensemble; the vertical line shows the value of the peak flow obtained by using the precipitation prediction provided by the LAM.

\subsection{Operational downscaling with RainFARM}

In this section we illustrate the application of RainFARM to the selected LAM event. The downscaling procedure preserves the large scale structure of the meteorological forecast and generates small-scale fields that are consistent with the LAM in terms of rainfall volume and spectral properties.

We choose $L_{0}=28 \mathrm{~km}$ (four times the model spatial resolution, see Patterson and Orszag, 1971) and we determine $T_{0}$ by considering a mean advection velocity $U_{0}=10 \mathrm{~km} / \mathrm{h}$; we obtain $T_{0} \simeq U_{0} / L_{0} \simeq 3 \mathrm{~h}$. The original LAM field is then aggregated at the spatial-temporal resolutions $\left(L_{0}, T_{0}\right)$. Notice that since $T_{0}=3 \mathrm{~h}$, which is the original LAM resolution, we do not perform any aggregation in time.

The downscaling procedure estimates the power spectrum of the aggregated LAM field and its spectral slopes. In this case we obtain $\alpha=2.35$ and $\beta=1.00$. These values are consistent with those derived from the analysis of precipitation fields measured by meteorological radars at midlatitudes (Veneziano et al., 1996; Menabde et al., 1999).

An ensemble of 100 spatial-temporal fields is then generated by following the procedure described in Sect. 2. These fields have a spatial resolution of $1.75 \mathrm{~km}$ and a time step of 10 min.

Before delving into the issue of probabilistic flood forecasting, we show a comparison between the original LAM field and one of the possible high-resolution precipitation fields generated by RainFARM. This comparison aims at illustrating some aspects of the downscaling procedure theoretically described in Sect. 2 . 


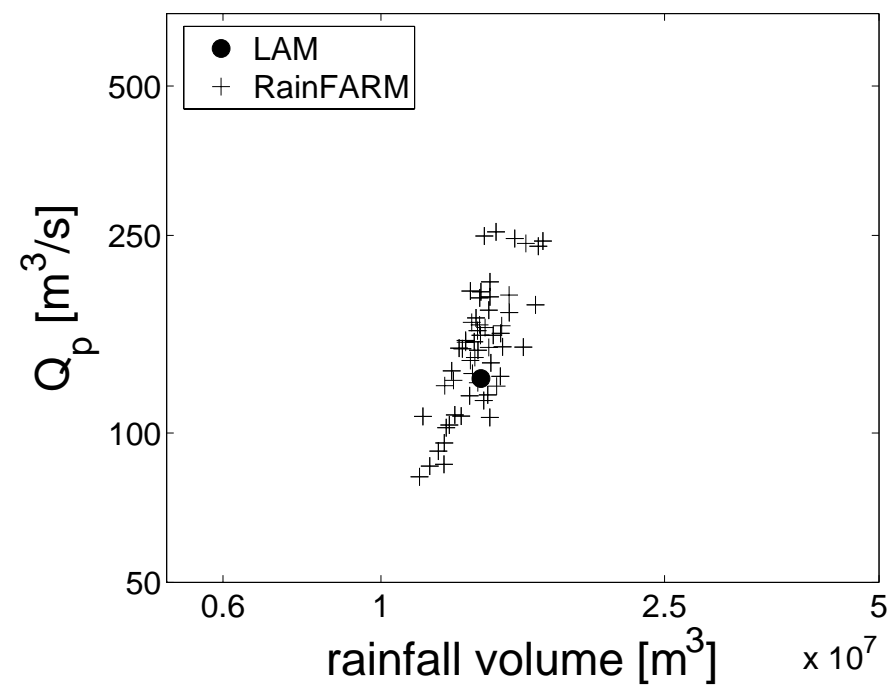

Fig. 9. Ensemble of 100 peak discharges for the Bisagno catchment $\left(A \simeq 100 \mathrm{~km}^{2}\right.$ ) versus the precipitation volumes that originate these peak values. The black point represents the deterministic value obtained by using the LAM prediction while the crosses are the 100 values generated by the RainFARM ensemble.

Figure 3 shows the spatial power spectra of the original and of the downscaled fields. There is a very good agreement between the two spectra for wavenumbers smaller than $1 / L_{0}$. At smaller scales, the LAM spectrum fails to display the power-law behavior that characterizes observed precipitation fields, while the spectrum of the downscaled field is much more consistent with the behavior obtained from meteorological radars at such scales.

We next consider the temporal evolution of the rainfall field. As an illustration, figure 4 a shows the evolution on a single continental pixel of precipitation downscaled to $10 \mathrm{~min}$ resolution. The spatially-averaged rainfall intensity is given by

$\bar{p}(t)=\langle p(x, y, t)\rangle_{x y}=\frac{1}{N_{x} N_{y}} \sum_{x=1}^{N_{x}} \sum_{y=1}^{N_{y}} p(x, y, t)$.

where $p$ is a generic precipitation field ( $r$ or $P$ ), $N_{x}=N_{y}=256$ grid points for the downscaled field and $N_{x}=N_{y}=64$ grid points for the LAM prediction. Figure $4 \mathrm{~b}$ reports the resulting time series in both cases.

We define also the temporal averages:

$\bar{p}(x, y)=\langle p(x, y, t)\rangle_{t}=\frac{1}{N_{t}} \sum_{t=1}^{N_{t}} p(x, y, t)$

where $N_{t}=288$ for the RainFARM field, since each timestep of $3 \mathrm{~h}$ splits into 18 steps at a temporal resolution of $10 \mathrm{~min}$, while $N_{t}=16$ for the LAM forecasts. Figure 5 compares the resulting spatial fields.
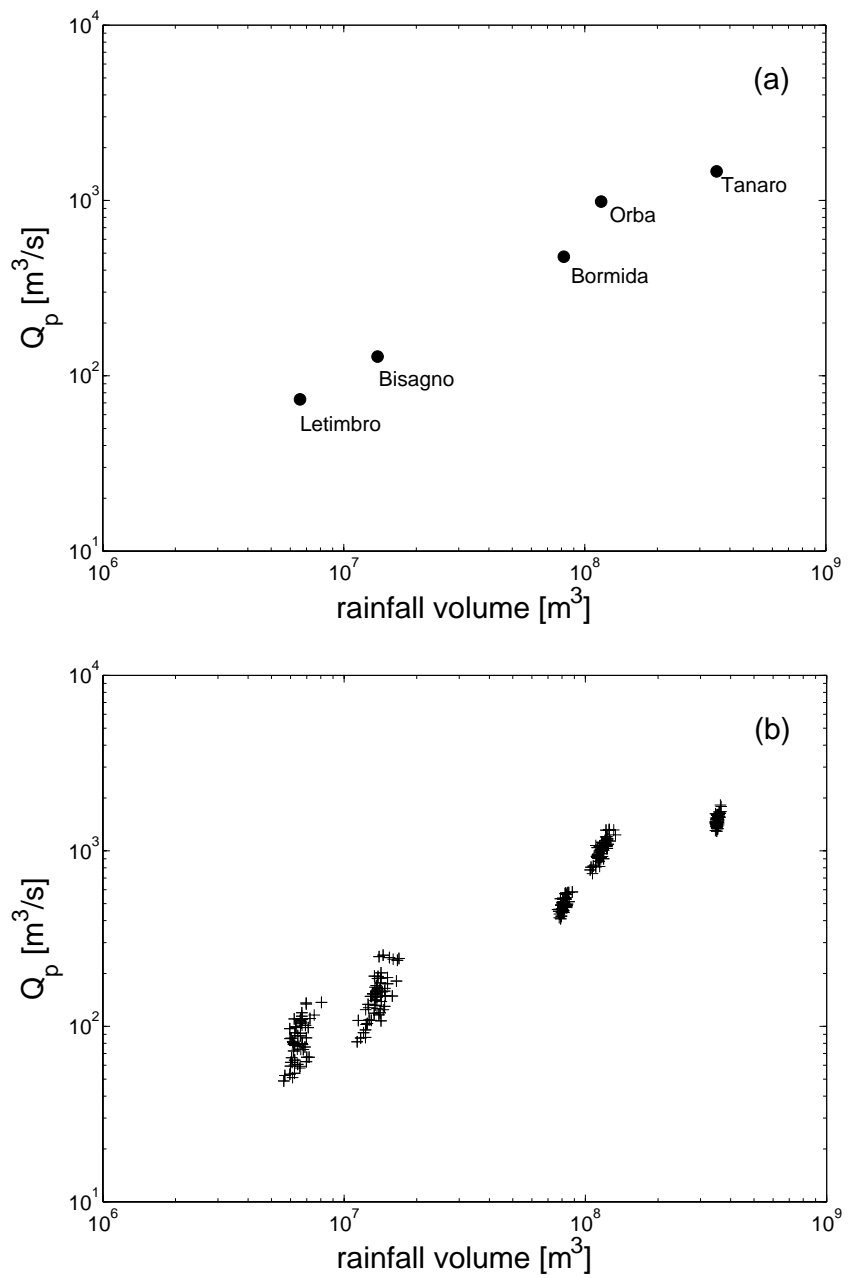

Fig. 10. Ensemble of 100 peak discharges for all the catchments considered in the analysis versus the precipitation volumes that originate these peak values. Panel (a) represents the deterministic values obtained by using the LAM prediction; the values refer, from left to right, to rivers Letimbro $\left(A \sim e q 30 \mathrm{~km}^{2}\right)$, Bisagno $(A \sim e q$ $\left.100 \mathrm{~km}^{2}\right)$, Bormida di Millesimo $\left(A \sim e q 250 \mathrm{~km}^{2}\right)$, Orba $(A \sim e q$ $\left.800 \mathrm{~km}^{2}\right)$ and Tanaro closed at Alba $\left(A \sim e q 3500 \mathrm{~km}^{2}\right)$. Panel (b) shows the probabilistic values obtained by applying the stochastic downscaling approach.

In both cases, the graphical comparison shows that the downscaling procedure is able to generate sub-grid fluctuations while preserving the large-scale features of the LAM field, such as the position of the rainfall patterns over the Alps and the Apennines.

In Fig. 6 we show an example of spatial downscaling, panels (b)-(e), compared to the corresponding large-scale prediction panel (a). Since the downscaling is performed both in space and time, Figs. 6(b)-(e) represent downscaled fields averaged at the LAM temporal resolution.

The RainFARM stochastically increases the resolution of the LAM prediction by creating rainfall fluctuations at the scale of hydrological processes. These stochastic predictions 


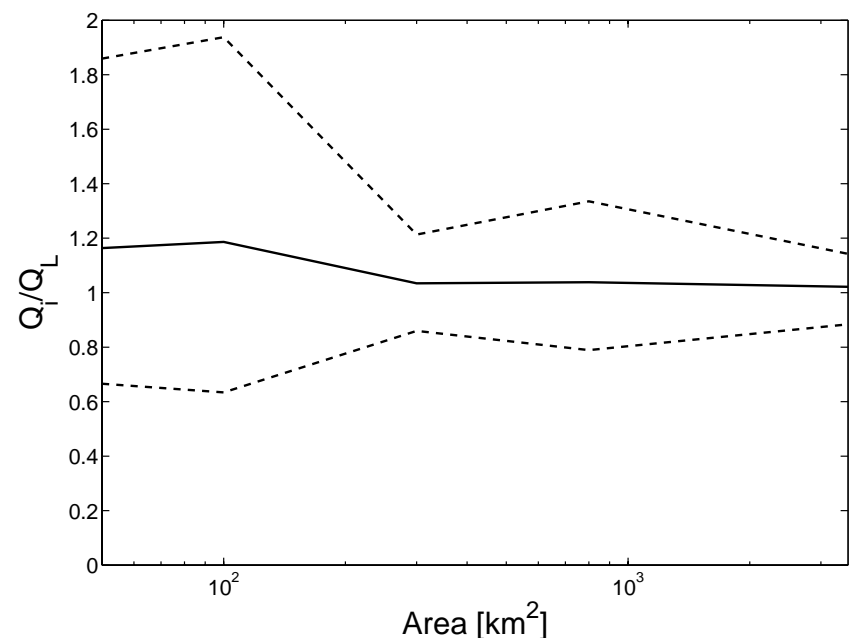

Fig. 11. Ratio between stochastic and deterministic peak discharge. The continuous line represents the average value and the dashed lines are the $95 \%$ confidence intervals.

are used for generating ensemble flood forecasts in several catchments within the downscaling domain.

\subsection{Ensemble discharge forecasting}

To perform a probabilistic discharge forecast we feed the ensemble of high-resolution fields provided by RainFARM to the semi-distributed hydrological model DRiFt (see Giannoni et al. (2005) and references therein for a complete description).

DRiFt is based on a geomorphologic approach and it has been designed to reproduce correctly the peak discharge value and its time of occurence. It accounts for the spatial variation of inputs such as rainfall and considers distributed morphologic and geological characteristics of the basin, using lumped parameters. For these reasons, DRiFt compounds most of the advantages of both distributed and lumped models.

In this experiment we consider five catchments located in the Apennines between Liguria and Piedmont. The basins considered are: (1) Letimbro $\left(A \simeq 30 \mathrm{~km}^{2}\right)$; (2) Bisagno $\left(A \simeq 100 \mathrm{~km}^{2}\right)$; (3) Bormida di Millesimo $(A \simeq$ $\left.250 \mathrm{~km}^{2}\right)$; (4) Orba $\left(A \simeq 800 \mathrm{~km}^{2}\right)$; (5) Tanaro closed at Alba $\left(A \simeq 3500 \mathrm{~km}^{2}\right)$.

The use of a deterministic precipitation prediction generates a single hydrograph, indicated by the white dashed line in Fig.7. If we use the RainFARM outputs as input for the DRiFt model we obtain an ensemble of 100 hydrographs, represented in Fig. 7 by the black continuous lines .

Each small scale evolution of the precipitation field is an independent realization of the same process. Therefore, each peak discharge value has a probability of occurrence equal to $1 / 100$. The probability of exceedence $(\operatorname{Pr}[Q>q])$ of a generic threshold value $q$ for the peak discharge in the Bis- agno river is plotted on a Gumbel chart (Fig. 8). The black points represent peak discharge data generated by the downscaled ensemble. The vertical line in the plot denotes the value of the peak flow obtained for the deterministic LAM prediction; we can observe that it shows a probability of being exceeded $\operatorname{Pr}\left[Q>Q_{L}\right] \simeq 0.7$.

We next consider the spread of peak discharge versus the spread of the corresponding rainfall volume. Figure 9 shows this analysis for the Bisagno river. The deterministic value obtained from the LAM prediction is compared with the ensemble of realizations of the probabilistic prediction. Notice how strong fluctuations in the peak discharge are observed also for events whose precipitation volume is very similar to the LAM forecast volume. This confirms that the sub-grid distribution of precipitation volumes can be crucial for assessing the uncertainty associated with flood prediction.

Similar results are obtained for all the catchments considered in our experiment (Fig. 10). The results indicate the presence of scale dependence in the amplitude of the peak discharge distribution. Moving from small basins (Letimbro and Bisagno) to the larger ones (Bormida and Orba) the spread in peak discharge decreases until, at the scale of the Tanaro basin, it is mainly due to fluctuations in the total precipitation volume. A more detailed exploration of how the variability of the hydrological response depends on the gap of scales between meteorological inputs and hydrological processes is discussed in Gabellani et al. (2006).

Fig. 11 shows the distribution of the ratio between stochastic and deterministic peak discharge values, $Q_{i} / Q_{L}$, as a function of basin area, for the 100 discharge values obtained above. For small catchments $\left(A<300 \mathrm{~km}^{2}\right)$ the subgrid fluctuations in the precipitation field generate a peak discharge distribution that has an uncertainty comparable to the value of the deterministic discharge prediction. For these basins, the average value of the stochastic peak discharge is $20 \%$ larger than the deterministic one, showing that the response of small catchments can be sensitive to small scale fluctuations in the rainfall field. For larger basins, no significant change in the average values is observed, but the stochastic approach is still useful for evaluating the uncertainty associated with the high-resolution precipitation prediction. In this case the variability of the peak discharge represents $20 \%-$ $30 \%$ of the deterministic value.

\section{Conclusions}

In this work we show an application of RainFARM, a new rainfall downscaling model based on nonlinearly filtering a random Gaussian process, which is capable of downscaling the large-scale information provided by meteorological models. This procedure represents a significant improvement over commonly available downscaling procedures used for operational purposes. It is able to conserve the total amount of precipitation predicted by the meteorological model, it 
takes into account anisotropy between space and time, conserves the correlations of meteorological rainfall fields both in space and in time and it preserves the position of large rainfall structures, to account for the effects of orography (Rebora et al., 2006). The features of the model make it suitable for operational applications. In particular this mode allows for a real-time estimate of the parameters by starting directly from the large scale rain field. In this way the model is self-consistent and it does not need calibration.

Coupling RainFARM outputs with a semi-distributed hydrological model allows for evaluating the use of this downscaling technique within a hydrometeorological forecasting chain. This study aimed at evaluating the benefits of applying RainFARM to the estimate of the uncertainty in flood predictions over small and medium Mediterranean catchments. Our results confirm the usefulness of adopting a downscaling technique for all the watersheds considered in this work. The downscaling procedure becomes crucial for basins with areas smaller than a few hundred square kilometers.

In this work we evaluated only the uncertainties in the peak discharge deriving from considering, in a probabilistic way, small-scale fluctuations in the precipitation field. A larger source of uncertainty is associated with the skill of numerical meteorological forecasts provided by LAMs. This issue can be addressed by using the output of global or local ensemble prediction systems in cascade with dowscaling procedures and rainfall-runoff models. We will consider the application of RainFARM in the framework of a meteorological ensemble prediction system in a future study.

Acknowledgements. We thank Tiziana Paccagnella and Chiara Marsigli at ARPA-SMR for providing us with the Lokal Model precipitation data.

Edited by: F. Guzzetti

Reviewed by: three referees

\section{References}

Bacchi, B., Ranzi, R., and Richard, E.: Hydrometeorological processes and floods in the Alps, Hydrol. Earth Syst. Sc., 7, 783948, 2003.

Bell, T. and Kundu, P.: Comparing satellite rainfall estimates with rain gauge data: optimal strategies suggested by a spectral model, J. Geophys. Res., 108 D3, 4121-4136, 2003.

Bell, T. L.: A space-time stochastic model of rainfall for satellite remote-sensing studies, J. Geophys. Res., 92D, 9631-9643, 1987.

Crane, R. K.: Space-Time structure of rain rate fields, J. Geophys. Res., 95(D3), 2011-2020, 1990.

Droegemeier, K. K., Smith, J. D., Businger, S., III, C. D., Doyle, J., Duffy, C., Foufoula-Georgiou, E., Graziano, T., James, L. D., Krajewski, V., LeMone, M., Lettenmaier, D., Mass, C., Sr., R. P., Ray, P., Rutledge, S., Schaake, J., and Zipserp, E.: Hydrological Aspects of Weather Prediction and Flood Warnings: Report of the Ninth Prospectus Development Team of the U.S., Weather Research Program, Bull. A. Met. Soc., 81(11), 2665-2680, 2000.
Ferraris, L., Rudari, R., and Siccardi, F.: The uncertainty in the prediction of flash floods in the northern mediterranean environment, J. Hydromet., 3, 714-727, 2002.

Ferraris, L., Gabellani, S., Parodi, U., Rebora, N., von Hardenberg, J., and Provenzale, A.: Revisiting Multifractality in Rainfall Fields, J. Hydromet., 4, 544-551, 2003a.

Ferraris, L., Gabellani, S., Rebora, N., and Provenzale, A.: A comparison of stochastic models for spatial rainfall downscaling, Water Resources Res., 39, 1368-1384, 2003b.

Gabellani, S., Boni, G., Ferraris, L., von Hardenberg, J., and Provenzale, A.: The propagation of uncertainty from rainfall to runoff: A simulation study, sub judice, 2006.

Giannoni, F., Roth, G., and Rudari, R.: A procedure for drainage network identification from geomorphology and its application to the prediction of the hydrologic response, Adv. Water Resour., 28(6), 567-581, 2005.

Guillot, G. and Lebel, T.: Disaggregation of Sahelian mesoscale convective system rain fields: Further developments and validation, J. Geophys. Res., 104, 31 533-31 551, 1999.

Lanza, L.: A Conditional Simulation Model of Intermittent Rain Fields, Hydrol. Earth Syst. Sci., 4, 173-183, 2000, http://www.hydrol-earth-syst-sci.net/4/173/2000/.

Lin, C. A., Wen, L., Béland, M., and Chaumont, D.: A coupled atmospheric- hydrological modeling study of the $1996 \mathrm{Ha}$ ! Ha! River basin flash flood in Québec, Canada, Geophys. Res. Lett., 29(2), 1026, doi:10.1029/2001GL013 827, 2002.

Matheron, G.: The intrinsic random functions and their application, Adv. Appl. Probab., 5, 439-468, 1973.

Menabde, M., Seed, A., Harris, G., and Austin, G.: Multiaffine random field model of rainfall, Water Resources Res., 35, 509514, 1999.

Patterson, G. and Orszag, S.: Spectral calculations of isotropic turbulence: efficient removal of aliasing interaction, Phys. Fluids, 14, 2538-2541, 1971.

Perica, S. and Foufoula-Georgiou, E.: Model for multiscale disaggregation of spatial rainfall based on coupling meteorological and scaling descriptions, J. Geophys. Res., 26, 347-361, 1996.

Press, W. H., Flannery, B. P., Teukolsky, S. A., and Vetterling, W. T.: Numerical Recipes in FORTRAN, Cambridge University Press, 1992.

Rebora, N., , Ferraris, L., von Hardenberg, J., and Provenzale, A.: Stochastic downscaling of LAM predictions: an example in the Mediterranean area, Adv. Geosciences, 2, 181-185. SRef-ID: 1680-7359/adgeo/2005-2-181, 2005.

Rebora, N., , Ferraris, L., von Hardenberg, J., and Provenzale, A.: The RainFARM: Downscaling LAM predictions by a Filtered AutoRegressive Model, J. Hydromet., in press, 2006.

Siccardi, F., Boni, G., Ferraris, L., and Rudari, R.: A reference framework for probabilistic flood forecast, J. Geophys. Res., 110, D05 101, doi:10.1029/2004JD005 314, 2005.

Veneziano, D., Bras, R., and Niemann, J.: Nonlinearity and selfsimilarity of rainfall in time and a stochastic model, J. Geophys. Res., 101 D, 26371-26392, 1996.

Venugopal, V., Foufoula-Georgiou, E., and Sapozhnikov, V.: A space-time downscaling model for rainfall, J. Geophys. Res., 104, 19705-19721, 1999. 